\title{
Lung epithelial cells: therapeutically inducible effectors of antimicrobial defense
}

\author{
MM Leiva-Juárez ${ }^{1}, \mathrm{JK}_{\text {Kolls }}^{2}$ and SE Evans ${ }^{1,3}$
}

Lung epithelial cells are increasingly recognized to be active effectors of microbial defense, contributing to both innate and adaptive immune function in the lower respiratory tract. As immune sentinels, lung epithelial cells detect diverse pathogens through an ample repertoire of membrane-bound, endosomal, and cytosolic pattern-recognition receptors (PRRs). The highly plastic epithelial barrier responds to detected threats via modulation of paracellular flux, intercellular communications, mucin production, and periciliary fluid composition. Epithelial PRR stimulation also induces production of cytokines that recruit and sculpt leukocyte-mediated responses, and promotes epithelial generation of antimicrobial effector molecules that are directly microbicidal. The epithelium can alternately enhance tolerance to pathogens, preventing tissue damage through PRR-induced inhibitory signals, opsonization of pathogen-associated molecular patterns, and attenuation of injurious leukocyte responses. The inducibility of these protective responses has prompted attempts to therapeutically harness epithelial defense mechanisms to protect against pneumonias. Recent reports describe successful strategies for manipulation of epithelial defenses to protect against a wide range of respiratory pathogens. The lung epithelium is capable of both significant antimicrobial responses that reduce pathogen burdens and tolerance mechanisms that attenuate immunopathology. This manuscript reviews inducible lung epithelial defense mechanisms that offer opportunities for therapeutic manipulation to protect vulnerable populations against pneumonia.

\section{INTRODUCTION}

The lung epithelium has long been perceived as a passive conduit for bulk airflow or an inert barrier to gas exchange, seldom encountering microbes and irrelevant to hostpathogen interactions. However, modern molecular techniques have revealed the complexity of the lower respiratory tract microbiome ${ }^{1}$ and accumulating evidence demonstrate that lung epithelial cells function as important mediators of host defense. ${ }^{2}$ Lung epithelial cells express an expansive complement of pattern-recognition receptors (PRRs) with oligospecificity for conserved microbial and host motifs. PRR activation by pathogen-associated molecular patterns (PAMPs) or danger-associated molecular patterns (DAMPs) initiates signaling cascades that can promote pathogen exclusion or expulsion, recruit and activate leukocyte-mediated defenses, directly kill microbes, and restore host homeostasis. These varied mechanisms provide manifold theoretical opportunities for intervention, and recent studies confirm that epithelial defenses can be therapeutically manipulated to protect the host, even in the setting of immunosuppression or leukodepletion. ${ }^{3}$ This review addresses important lung epithelial pathogen detection and response mechanisms that may be therapeutically manipulated to prevent and treat lower respiratory tract infections in healthy and immunocompromised populations.

\section{INDUCIBLE BARRIER DEFENSES}

Cellular junctions and cytoskeletal elements

The histological complexity of the lung epithelium portends the specialized functions of its component cells. The pseudostratified airway epithelium is predominantly comprised of ciliated cells and secretory cells, interspersed with regenerative basal cells and neuroendocrine cells (Figure 1a,b). The vast majority of the alveolar epithelial surface area is contributed by exceptionally thin, broad type I pneumocytes that are optimized for gas exchange, while the considerably more numerous type II pneumocytes are principally responsible for

${ }^{1}$ Department of Pulmonary Medicine, The University of Texas MD Anderson Cancer Center, Houston, Texas, USA. ${ }^{2}$ Center for Translational Research in Infection and Inflammation, Tulane School of Medicine, New Orleans, Louisiana, USA and ${ }^{3}$ The University of Texas Graduate School of Biomedical Sciences, Houston, Texas, USA. Correspondence: SE Evans (seevans@mdanderson.org) 


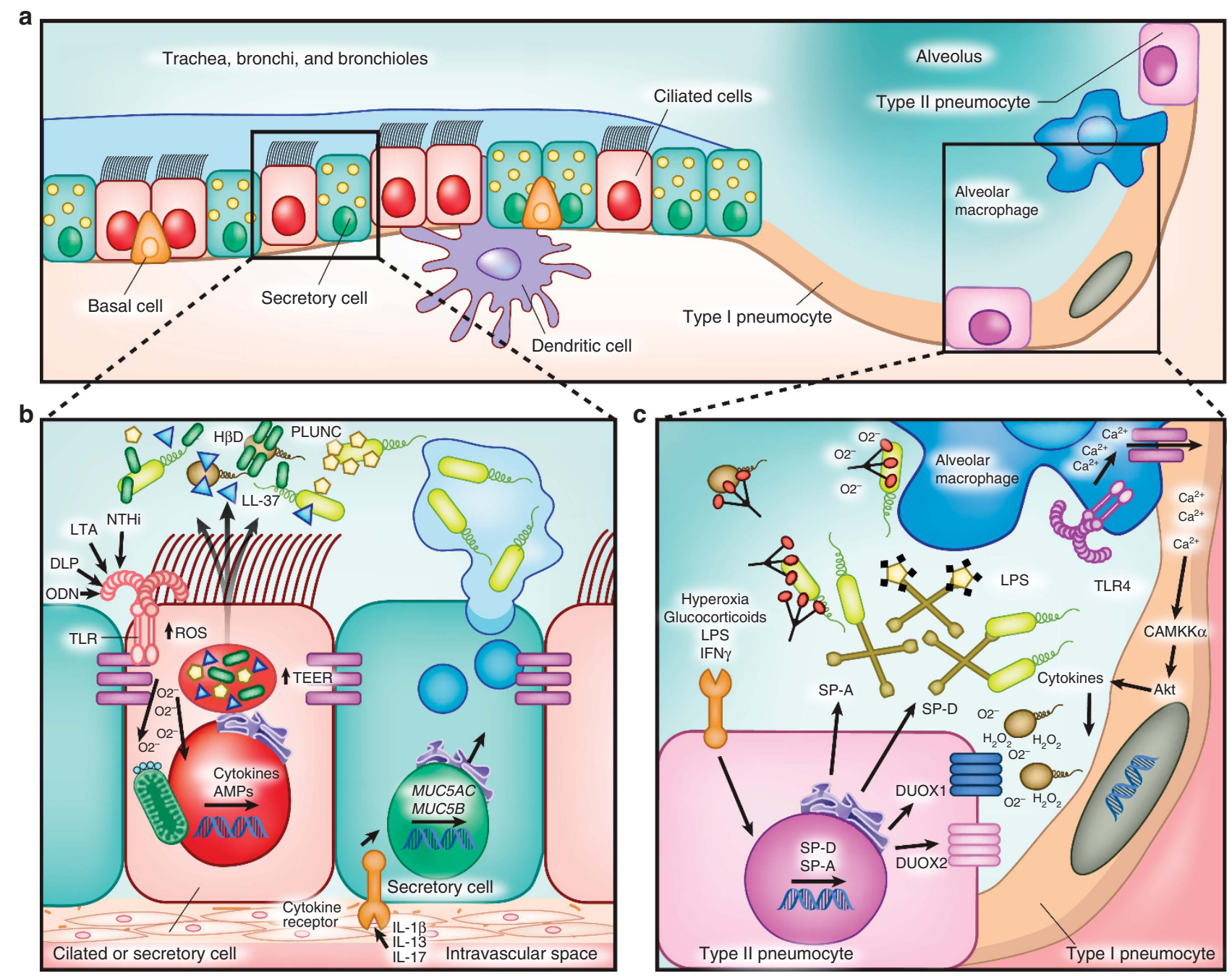

Figure 1 Inducible antimicrobial resistance mechanisms of lung epithelial cells. (a) Cells contributing to inducible epithelial airspace defense. (b) Inducible responses in the conducting airways. Pattern-recognition and cytokine receptors detect local danger signals in the conducting airways, responding with enhanced barrier and mucociliary functions to improve pathogen exclusion, increased production of microbicidal antimicrobial peptides and volatile species, and secretion of mediators of leukocyte recruitment and activation. (c) Inducible responses in the alveolar compartment. Epithelial cells in the gas exchange units of the lungs detect pathogen-associated molecular patterns, perceive stress signals and communicate with lung resident leukocytes, and respond through inducible modulation of barrier function, enhanced production of antimicrobial peptides, collectins and volatile species, and secretion of leukocyte-active cytokines. LTA, lipotechtoic acid; DLP, diacylated lipopeptides; H $\beta D$, human $\beta$-defensin; NTHi, non-typeable Haemophillus influenza; ODN, oligodeoxynucleotide; ROS, reactive oxygen species; SP, surfactant protein; TLR, Toll-like receptor; TEER, transepithelial electrical resistance.

secretory functions of the peripheral lung ${ }^{4}$ (Figure 1a,c). At baseline, these epithelial populations form a continuous $100 \mathrm{~m}^{2}$ barrier interface between the host and the external environment. Following PRR activation, this barrier function can be actively adapted to enhance microbial protection. Tight and adherens junctions, connecting cytoskeletons of apposing cells, modulate paracellular flux of ions and macromolecules through structural protein phosphorylation or translation of alternate tight junction protein isoforms to prevent barrier disruption and lung injury in both the airways and alveolar space. ${ }^{5,6}$ Inducible modification of paracellular permeability regulates access to epithelial receptors for PAMPs, cytokines, and intraepithelial leukocytes. ${ }^{7,8}$ Epithelial tight junctions also contain many potentially targetable signaling molecules, including protein kinase $\mathrm{C}$, Rho proteins, phosphatidyl inositol 3-kinase, transcription factors, and epidermal growth factor receptor family members, such as HER2/3. ${ }^{7,8}$ For example, Toll-like receptor (TLR)-2 stimulation of bronchial epithelial cells can activate $\mathrm{PKC} \zeta$, increasing claudin-1 expression, thereby enhancing transepithelial electrical resistance and tight junctional integrity. ${ }^{9}$ Epithelial cytoskeletons can also rearrange to facilitate PRR signaling, and cytoskeletal elements themselves can augment host defenses, as when F-actin released during necrosis activates dendritic cell CLEC9A receptors ${ }^{10}$ promoting clearance of dying cells and, ultimately, hastening resolution of infection-related injury.

Perhaps underscoring the importance of these responses, several bacteria possess the ability to enter non-phagocytic cells 
via cytoskeletal manipulation and form sequestering compartments that promote their own growth. Mitigating this threat, mammalian PRRs such as NOD1, NOD2, and pyrin can sense pathogenic cytoskeletal protein changes, leading to initiation of proinflammatory responses and entrapment of actin-polymerizing bacteria by septin cages. ${ }^{11}$ Thus, although not yet established in practice, manipulation of junctional and cytoskeletal elements may eventually provide valuable opportunities to protect against respiratory infections.

\section{Mucociliary defenses}

Particles and microbes entrained into the lower respiratory tract during respiration are impacted into the airway lining fluid by turbulent flow and $>90 \%$ are expelled from the lungs via the mucociliary escalator within minutes. ${ }^{12}$ The largest populations of airway epithelial cells, present in roughly equal numbers, are the ciliated cells that beat the airway lining fluid proximally toward the glottis and the secretory cells that, among other things, contribute mucins to the airway lining (Figure 1b). MUC5AC and MUC5B are the most abundantly expressed polymeric secreted mucins in the airways. ${ }^{13}$ In addition to optimizing the viscoelastic properties to the airway lining fluid for particle clearance, there is mounting evidence that gel-forming mucins contribute to antimicrobial responses. Stimulated MUC5B production from epithelial cells has been directly linked to antibacterial defense and enhanced alveolar macrophage function, ${ }^{12}$ and is inducible by PAMPs such as $\beta$ glucan $^{14}$ and by some NF- $\kappa$ B-mediated cytokines (e.g., IL-1 $\beta$, IL-17A) ${ }^{15}$ Concordantly, MUC5B-deficient mice have been found to be hypersusceptible to pneumonia and to demonstrate impaired pathogen clearance. ${ }^{12}$ MUC5AC is strongly induced by epithelial exposure to IL-13, cathelicidin, and a number of bacterial and viral pathogens, ${ }^{12,16-18}$ including specificity protein 1 (Sp1)- and epidermal growth factor receptormediated induction by influenza A. ${ }^{19}$ Cytosolic PRR stimulation may also enhance airway mucin production, as NLRP6 inflammasome activation promotes mucin secretion during colonic bacterial infections, although this is not yet confirmed in the lung. ${ }^{20}$ Active epithelial regulation of periciliary layer $\mathrm{pH}$ further augments mucin-facilitated pathogen clearance and defense. The importance of this function is evident in cystic fibrosis, where reduced periciliary $\mathrm{pH}$ impairs detachment of submucosal mucins and impedes mucus clearance. ${ }^{21}$ The multiple stimuli capable of inducing MUC5AC and MUC5B secretion suggest their potential as therapeutic targets. While membrane-bound mucins (MUC1, MUC4, MUC16) also facilitate mucociliary escalator function, there is limited evidence that their induction by infectious or pharmacological stimuli enhances pathogen clearance. ${ }^{13}$ No strategies have definitively shown improved pathogen clearance through ciliary function manipulation in hosts without intrinsic impairments. However, recent data revealing that IL-13induced bronchial epithelial cell MUC5AC contributes to mucostasis in asthma via tethering of mucus gel domains to the epithelium $^{22}$ suggest there may be additional opportunities to augment mucociliarly pathogen clearance in patients with baseline defects, beyond inducing mucus production and moderating periciliary $\mathrm{pH}$.

\section{PATHOGEN DETECTION}

PRRs are highly conserved across species, emphasizing their importance to host survival. $^{23-25}$ The broad spectrum of PRRs expressed by lung epithelial cells (Table 1) allow PAMP sensing within most cellular compartments, facilitating rapid responses to infections, and offering abundant opportunities for therapeutic elicitation of protective epithelial functions.

\section{Toll-like receptors}

Lung epithelial cells are reported to express all known human TLRs, including those that localize to the plasma membrane (TLR2/1, TLR2/6, TLR4, TLR5, TLR10) and those that localize to endosomes (TLR3, TLR7, TLR8, TLR9). ${ }^{26} \mathrm{~N}$-terminal leucine-rich repeat (LRR) domains with varying degrees of $\mathrm{N}$-glycosylation form a characteristic TLR solenoid structure responsible for pathogen sensing. ${ }^{27}$ A single transmembrane domain connects leucine-rich repeat domains to a cytoplasmic Toll/IL-1 receptor (TIR) C-terminus tail that recruits TIR adaptor proteins to initiate downstream signaling. Despite the structural similarity of TLRs, their ligand specificity and binding sites vary substantially, allowing them to detect a remarkable spectrum of patterns, including such diverse moieties as lipopeptides, nucleic acids, and bacterial proteins, making TLRs frequent targets of studies investigating epithelial manipulation strategies, as outlined below.

\section{NOD-like receptors}

NOD-like receptors (NLRs) are soluble cytosolic proteins composed of a C-terminal leucine-rich repeat domain that confers ligand specificity, a central nuclear oligomerization domain (NOD) and a variable N-terminal effector domain. ${ }^{28}$ They are further categorized by their effector domains into caspase recruitment domain (CARD or NLRCs), pyrin domain (PYD or NLRPs) or baculoinhibitor of apoptosis protein domain (NAIPs or NLRBs). The active oligomerization of NLRs, adaptor proteins and caspases comprises inflammasome formation, resulting in cleavage of pro-IL-1 $\beta$ and pro-IL-18 into their active forms. ${ }^{29}$

NLRs expressed by lung epithelial cells include NOD1 and NOD2 which bind bacterial peptidoglycan moieties, activating mitogen-activated protein (MAP) kinase, NF- $\kappa \mathrm{B}$, and autophagic pathways. ${ }^{3}$ Deficiency ${ }^{30}$ or polymorphisms ${ }^{31}$ of these receptors results in increased susceptibility to respiratory infections. NLRP1 enhances resistance to pneumonia by detecting virulence factors such as Bacillus anthracis lethal toxin. ${ }^{32}$ NLRP3 is an important bronchial epithelial DAMP sensor during infection, ${ }^{33,34}$ detecting potassium efflux, ${ }^{35}$ excess reactive oxygen species (ROS) production, ${ }^{36}$ or mitochondrial dysfunction. ${ }^{37}$ NLRC4 initiates inflammasome formation following detection of flagellated bacteria such as Legionella pneumophila, ${ }^{38}$ P. auruginosa, ${ }^{39}$ Klebsiella pneumoniae, and those expressing type-III secretion systems (T3SS). NLRX1 possesses an N-terminal mitochondrial targeting sequence and appears to contribute to ROS production by 
Table 1 PRRs in the pulmonary epithelium

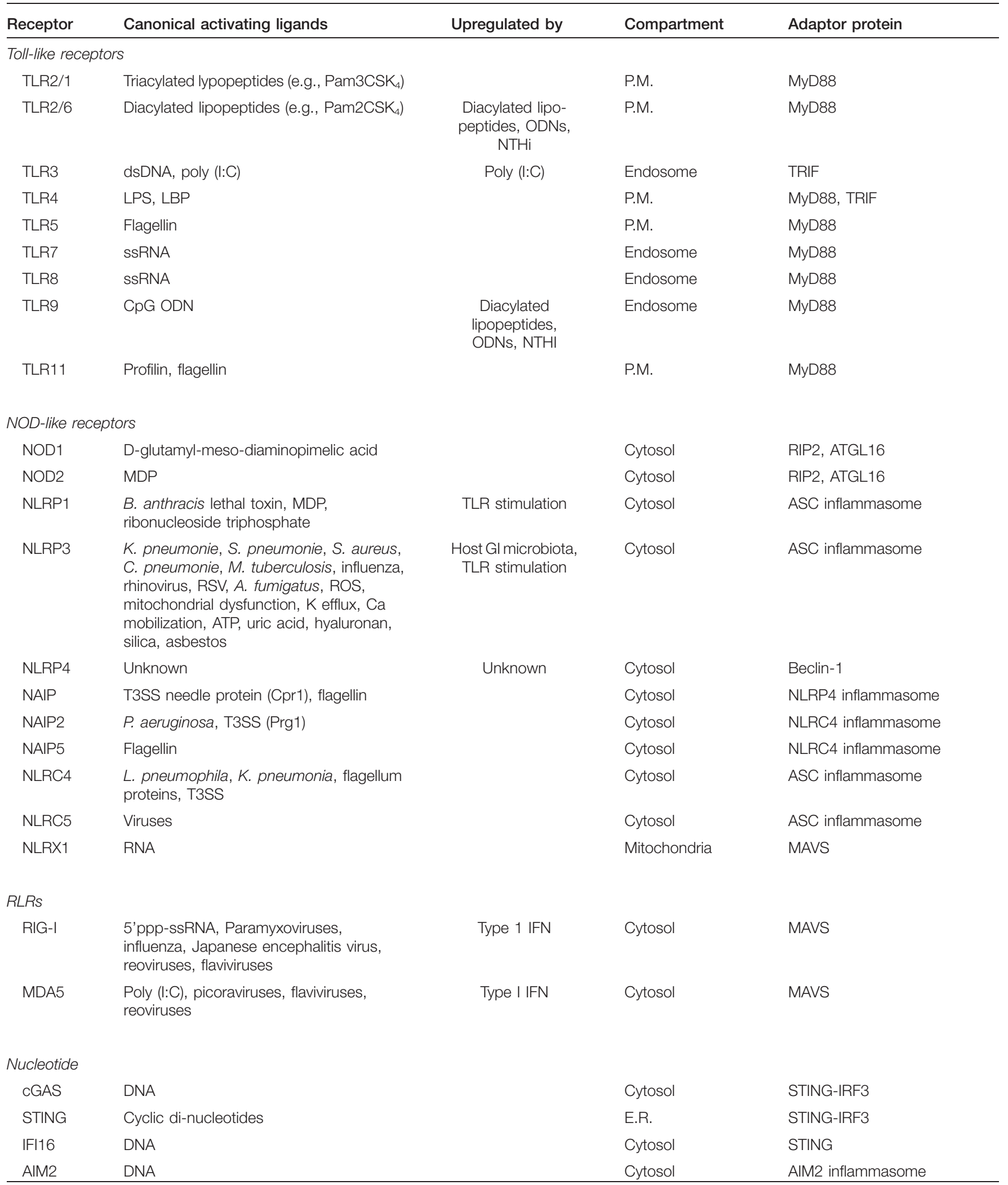

ATP, adenosine triphosphate; E.M., endoplasmic reticulum; Gl, gastrointestinal; LBP, lipopolysaccaride-binding protein; LPS, lipopolysaccharide; MDP, muramyl dipeptide; NTHi, non-typeable Haemophilus influenza; ODN, oligodeoxynucleotide; P.M., plasma membrane; Poly I:C: poly-inosine:poly-cytosine; RLRs, Rig-I-like receptors; ROS, reactive oxygen species; RSV, respiratory syncitial virus; TLR, toll-like receptor; TTSS, type three secretion system. 
the respiratory chain transport when stimulated by RNA. ${ }^{28}$ In contrast, stimulation of other lung epithelial NLRs, such as NLRC3 and NLRC5, is associated with anti-inflammatory responses, ${ }^{40}$ suggesting that therapeutic targeting of NLRs may allow selective activation or resolution of antimicrobial responses.

\section{Nucleic acid sensors}

Microbe- or host-derived nucleic acids, respectively, can serve as PAMPs or DAMPs that initiate antimicrobial responses. In addition to endosomal TLRs, multiple cytosolic receptors detect RNA and DNA in lung epithelial cells. ${ }^{41}$ This prominently includes retinoic acid inducible gene-I (RIG-I) and melanoma differentiation-associated gene 5 (MDA5), cytosolic RIG-I-like helicases (RLRs) that sense ssRNA and dsRNA with a 5 ' triphosphate motif. ${ }^{29}$ RLR expression is enriched in lung epithelial cells in response to type I interferons $^{42}$ and signal through the mitochondrial membrane-bound protein MAVS (also known as IPS-1, VISA, and Cardif) to elicit IRF3, IRF7, and NFKB mediated antimicrobial responses. RLR-MAVS signaling is critical to interferon production during RSV pneumonia, ${ }^{43}$ for example, and contributes to infection-induced airway hyperreactivity through increases in metalloproteinase and cathepsin production. ${ }^{44}$ Though less studied, lung epithelial MDA5 may also elicit virus-specific protection in other infection models. ${ }^{45}$

Independent of TLRs, NLRs and RLRs, lung epithelial cytosolic detection of microbial DNA and cyclic di-nucleotides $^{42}$ frequently involves signaling via stimulator of interferon genes (STING), an endoplasmic reticulum-localized protein that acts both as a direct receptor for bacteria-produced cyclic dinucleotides and as an adaptor molecule for other cytosolic nucleic acid receptors, ${ }^{46,47}$ such as IFI $16^{48}$ or DDX41 (which can also detect both DNA and cyclic dinucleotides). ${ }^{49,50}$ Additionally, 2'3'-cyclic GMP-AMP (cGAMP) is produced by cGAMP synthase (cGAS) in lung cells upon cGAS binding of microbial DNA, resulting in alternate activation of STING by cGAMP ${ }^{51}$ making both STING and cGAS frequent targets of investigation for potential therapeutic manipulation of responses from the lungs and elsewhere. Other STINGindependent lung epithelial DNA sensors such as DAI, AIM2 and LRRFIP $1^{\text {ref. } 52}$ have been described, although their relevance in inducible lung immunity remains less established.

\section{C-type lectins}

Pathogen-derived carbohydrates, including mannose, fucose, and glucans, can be detected by plasma membrane localized C-type lectins. These receptors are widely distributed on epithelial and myeloid cells and allow detection of fungi, viruses, and bacteria. ${ }^{53}$ Dectin-1 is expressed in bronchial epithelial cells and mediates inflammatory responses to infections caused by non-typeable Haemophilus influenza $(\mathrm{NTHi})^{54}$ and a number of fungi. ${ }^{55}$ Surfactant proteins SPA and SP-D also contain C-type lectin domains, and broadly contribute to host defense via pathogen detection, ${ }^{56}$ in addition to the antimicrobial effects discussed below. Epithelial cells can also detect fungal glucans via the non-lectin containing glycosphingolipid lactosylceramide. ${ }^{57}$

\section{ANTIMICROBIAL EFFECTOR MOLECULES}

Upon PRR activation, lung epithelial cells not only generate cytokine signals that initiate leukocyte-mediated responses but they also produce numerous effector molecules that exert directly microbicidal effects (Table 2 and Figure 1) and have been targeted for potential therapeutic induction.

\section{Cathelicidin and defensins}

Small cationic antimicrobial proteins (AMPs) are frequently synthetized as pre-pro-peptides that are cleaved before secretion to expose share positively charged moieties that disrupt negatively charged microbial membranes, ${ }^{58,59}$ as is the case for lung epithelial defensins and cathelicidin. ${ }^{60}$ Defensins are amphipathic molecules that a common $\gamma$-core region and are further subdivided into $\alpha$-defensins and $\beta$-defensins based on disulfide bridge arrangement. Although previously thought to express only $\beta$-defensins, recent data indicate that murine lung epithelial cells also produce $\alpha$-defensin $5^{\text {ref. } 61}$ and $\alpha$ defensin 1 following exposure to bacterial lysates. ${ }^{62}$ The only human cathelicidin, hCAP18, possess two characteristic hydrophobic $\alpha$-helix domains. ${ }^{63}$ hCAP18 is stored in preformed granules and, upon stimulation, is cleaved on its $\mathrm{N}$-terminal domain to its active form, LL37. ${ }^{3}$ LL37 is secreted by airway and submucosal gland epithelial cells and is believed to electrostatically disrupt bacterial membranes. ${ }^{64}$ The functions of epithelial defensins and LL-37 are multiple, both selectively promoting and attenuating different elements of the inflammatory response. ${ }^{60,65}$ When induced by infections, both act as chemoattractants for neutrophils, dendritic cells, $\mathrm{T}$ cells, macrophages, and monocytes. ${ }^{66-68}$ LL-37 also interacts synergistically with IL-1 $\beta$ to promote local inflammatory cytokine production. ${ }^{69}$ However, its binding of negatively charged molecules also allows LL-37 to sequester lipopolysaccaride (LPS) and certain DAMPs (e.g., self-DNA and RNA), thereby attenuating other inflammatory responses. ${ }^{70-72}$

\section{Antiproteases}

Lung cells express secretory leukocyte protease inhibitor and elastin-specific inhibitor (elafin) in response to stimuli such as LPS, IL-1 $\beta$ and TNF. ${ }^{73}$ Secretory leukocyte protease inhibitor is expressed in the airway mucosa ${ }^{60}$ and inhibits neutrophil elastase, chymotrypsin, and cathepsin G, but notably also exerts directly antimicrobial effects on bacteria (Pseudomonas aeruginosa, Stephylococcus aureus, Escherichia coli), fungi (Candida albicans, Aspergillus fumigatus) and $\mathrm{HIV}{ }^{74,75}$ Similarly, elafin has direct antimicrobial activity against $P$. aeruginosa and S. aureus. ${ }^{76}$ Further, both of these antiproteases demonstrate immunomodulatory activity on macrophages and endothelial cells following epithelial stimulation, decreasing I $\mathrm{B} \alpha$ degradation $^{77}$ and increasing production of TGF $\beta$ and IL$10 .^{78}$ Properties promoting tissue repair and resolution of inflammation have also been attributed to secretory leukocyte protease inhibitor and elafin. ${ }^{79}$ 
Table 2 Inducible antimicrobial peptides in the lung

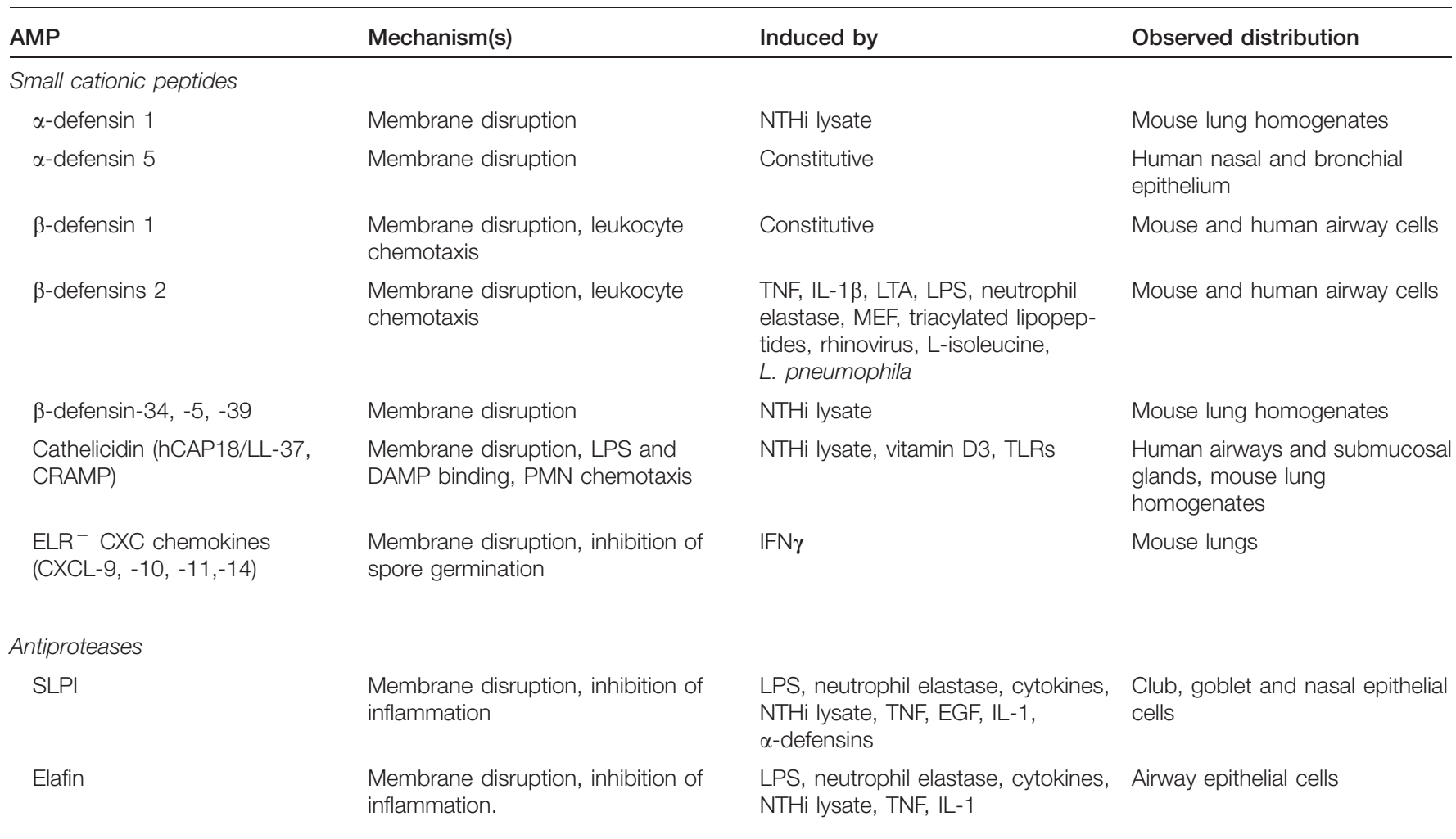

Iron modulators

$\begin{array}{ll}\begin{array}{l}\text { Lactoferrin } \\ \text { Lipocalin } 2\end{array} & \begin{array}{l}\text { Iron sequestration } \\ \text { Iron siderophore binding }\end{array} \\ \begin{array}{l}\text { Other AMPs } \\ \text { Lysozyme }\end{array} & \begin{array}{l}\beta_{1-4} \text { glycoside hydrolysis of } \\ \text { peptidoglycans }\end{array} \\ \text { Collectins (e.g., SP-A, SP-D) } & \begin{array}{l}\text { Opsonization, membrane } \\ \text { permeabilization }\end{array} \\ \text { SPLUNC1 (BPIFA1) } & \text { LPS and biofilm inhibition }\end{array}$

NTHi lysate

Submucosal glands

IL-22, NTHI lysate, LPS, IL-1B, IL-17 Airway epithelial cells

AMP, antimicrobial peptide; BPIFA1, BPI fold containing family A member 1; CRAMP, mouse cathelicidin-related antimicrobial peptide; ELR-, not containing glutamate-leucinearginine sequence; LPS, lipopolysaccharide; LTA, lipotechoic acid; MEF, myeloid ELF-1-like factor; NTHi, non-typeable Haemophilus influenzae; SLPI, secretory leukocyte protease inhibitor; SP, surfactant protein; SPLUNC1, short palate, lung, and nasal epithelial clone 1; TLR, Toll-like receptor; TNF, tumor necrosis factor.

\section{Chemokines}

Cytokine and chemokine production are essential to epithelial modulation of immune responses, but some IFN $\gamma$-induced epithelial chemokines, including CXCL9, CXCL10, and CXCL11, also demonstrate directly bactericidal effects on $B$. anthracis, E. coli, and Listeria monocytogenes, ${ }^{80,81}$ likely via membrane disruption, given their defensin-like $\alpha$-helical domains. The N-terminal domain of CXCL14 also appears to affect the integrity of Gram-positive and Gram-negative bacterial membranes. ${ }^{82}$

\section{Lysozyme, lactoferrin, and lipocalin 2}

Human lysozyme and mouse lysozyme- 1 and -2 are abundantly expressed by lung airway and submucosal gland epithelial cells, ${ }^{83}$ catalyzing hydrolysis of $\beta_{1-4}$ glycosidic bonds in bacterial peptidoglycan. ${ }^{3}$ This effect is sufficiently robust that transgenic mice overexpressing lysozyme display decreased susceptibility to pneumonias caused by $P$. aureginosa and Group B Streptococci. ${ }^{84}$ Lactoferrin is a cationic glycoprotein that can bind both free iron and bacterial membrane components, ${ }^{60}$ impairing bacterial growth. While the isolated effects of epithelial lysozyme and lactoferrin are predominantly bacteriostatic at physiological concentrations, together they synergize to exert bactericidal effects. ${ }^{85}$ Lysozyme's activity on Gram-negative bacteria is enhanced by lactoferrin increasing the permeability of bacterial outer membranes, facilitating cleavage of peptidoglycan bonds in the periplasmic space. Consistent observations of this positive interaction have promoted numerous studies of therapeutic supplementation with combinations of recombinant antimicrobial effectors. 
Lipocalin 2 is a secreted protein that is also induced by DAMPs and cytokines in multiple cell types, including lung epithelial cells, ${ }^{6,86-88}$ and has been found to be important in host responses against $K$. pneumoniae and E. coli. Similar to lactoferrin, lipocalin 2 has the ability to sequester iron from siderophores, though it appears likely that additional ironindependent mechanisms also contribute to its antibacterial effect.

\section{Collectins}

Lung epithelial collectins (collagen-containing C-type lectins), prominently including SP-A and SP-D, function as soluble PRRs in the airspaces that polymerize into large scaffolds and opsonize or aggregate microbes. SP-A and SP-D expression by type II alveolar epithelial cells and club cells increases in responses to LPS, glucocorticoids, hyperoxia, bacterial lysates, and IFN $\gamma,{ }^{62,89}$ suggesting they may be accessible therapeutic targets. SP-A- and SP-D-mediated opsonization enhances microbial clearance by alveolar macrophages (Figure 1c), while their deficiency increases susceptibility to Group B Streptococci, Gramnegative bacteria, RSV, influenza A, and adenoviruses. ${ }^{90} \mathrm{SP}$ $\mathrm{A}$ and SP-D are also reported to disrupt bacterial membrane permeability of some Gram-negative bacteria. ${ }^{91}$

\section{PLUNC proteins}

Palate-lung-nasal-clone (PLUNC) family proteins are secreted by cells throughout the lung and nasal mucosa. SPLUNC1 is induced by TLR2 stimulation in a MAP kinase/AP-1 and NF- $\kappa B$-dependent matter. ${ }^{92}$ Their antimicrobial properties are thought to relate to their structural homology with LPS-binding proteins, though they may also function as surfactant proteins, and possibly inhibit biofilm formation. ${ }^{93}$ SPLUNC1 deficient mice are susceptible to $P$. aeruginosa and $K$. pneumoniae pneumonia, and demonstrate impaired production of proinflammatory cytokines and other AMPs. ${ }^{94,95}$

\section{Reactive oxygen species and ion transport}

ROS are increasingly recognized to function as direct antimicrobial effector molecules, most likely through lipid peroxidation of microbial membranes and DNA damage, in addition to their well-established roles as signaling molecules. Lung epithelial cells generate ROS both constitutively and inducibly, most abundantly as superoxide or hydrogen peroxide. While all NADPH oxidase (NOX) isoforms are reportedly found in the lungs, dual oxidases (DUOX) are the principal ROS generators of lung epithelial cells. DUOX1 produces a relatively consistent amount of ROS, though recent evidence indicates that this production can be moderately enhanced by IL- 4 and IL-13 exposure. ${ }^{96}$ In contrast, DUOX2dependent ROS production can be profoundly increased by activation of existing DUOX2 and increased DUOX2 and DUOXA2 transcription following exposure to cytokines such as IFN $\gamma,{ }^{96}$ an intervention that has been shown in vitro to reduce pathogen burdens. DUOX enzyme activity is regulated by calcium concentrations and they are predominantly expressed apically on ciliated airway epithelial cells and on type II alveolar epithelial cells, approximating the enzymes to microbes that gain access to the airspaces ${ }^{97}$ (Figure 1c). In addition to toxic effects of the volatile species directly on pathogens, submucosal epithelial cell-derived lactoperoxidase catalyzes a reaction between epithelial hydrogen peroxide and thiocyanate to form the highly microbicidal molecule hypothiocyanate.

As observed in cystic fibrosis, impaired anion transport due to cystic fibrosis transmembrane conductance regulator (CFTR) mutations causes decreased bicarbonate secretion, resulting in reduced periciliary $\mathrm{pH}$ and impaired AMP function. ${ }^{98}$ In addition, active epithelial ion transport is required to provide halide (chloride, bromide, iodide) and pseudohalide (thiocyanate) substrates for ROS-mediated antimicrobial defenses. ${ }^{99}$ Thus, investigators have variously proposed to stimulate DUOX activity in the lungs, modify ion channel activity, and enhance halide/pseudohalide availability as means to enhance antimicrobial defenses of the lungs.

\section{EPITHELIAL MODULATION OF IMMUNE RESPONSES AND TOLERANCE}

PRR elicited epithelial cytokines importantly participate in the recruitment and activation of leukocytes in the lung. Beyond simply increasing the number of leukocytes present, epithelial cell responses clearly modulate adaptive immune responses in the lung during infections.

\section{Modulation of inflammation}

Lung epithelial cells have long been recognized to demonstrate profound asthmatic/allergic phenotypes when exposed to IL-4, IL-5, IL-9, and IL-13. However, it is now evident that they are also capable of autonomously promoting or initiating type 2 inflammatory responses. A typical example of this is TLR4mediated detection of house dust mite antigen ${ }^{100}$ by club cells, resulting in allergic cytokine secretion and Th2 immune deviation. This epithelial exposure promotes production of IL33 , TSLP, and IL-25, stimulating DCs to subsequently activate type 2 innate lymphoid cells (ILC2), basophils, eosinophils and mast cells. ${ }^{101}$ Epithelial granulocyte macrophage colonystimulating factor (GM-CSF) is also secreted in response to allergens and induces maturation, proliferation, and activation of antigen presenting cells that drive type 2 responses. ${ }^{102}$ Infection-released DAMPs also contribute to epithelial type 2 immune deviation. Epithelial cells respond to uric acid and ATP that are released into the airway in response to allergens, further releasing IL-33 and TSLP. ${ }^{103,104}$ While type 2 deviation is often regarded as maladaptive (i.e., allergy) or relevant only to parasitic diseases, it has been shown that enhanced CCL8 production and neutrophil recruitment associated with type 2 responses augment clearance of $K$. pneumoniae. ${ }^{105}$ Type 1 and type 17 inflammation are more commonly regarded as protective against respiratory pathogens, and lung epithelial cells are analogously capable of deviating immune responses to these patterns, as well. For example, lung cells express IFN- $\Upsilon$ in response to mycobacterial and fungal infections, promoting protective type 1 inflammatory deviation ${ }^{106,107}$ Similarly, not only do lung epithelial cells produce brisk cytokine responses when exposed to IL-17A and IL-17F but also epithelial 
production of IL-6, TGF $\beta$, and IL-21 (also possibly IL-23) can enhance differentiation of Th17 cell differentiation ${ }^{108-111}$ sculpting local adaptive responses in response to PAMP exposure.

\section{Tolerance and downregulation of inflammation}

Host survival of pathogen challenges depends on two fundamental strategies: resistance and tolerance. Resistance relies upon reductions in the microbial burden to limit injury and preserve fitness. Alternately, tolerance promotes survival through host adaptations to limit the pathogen's ability to inflict damage or to reduce noxious and/or maladaptive responses associated with host defenses (immunopathology), rather than eliminating the pathogen. ${ }^{112}$ Literature describing infection tolerance in the lung remains limited, and studies describing therapeutic manipulation are rarer still. However, induction of host-protective responses, rather than pathogentoxic elements, holds promise as a strategy that could be applicable in many infectious or inflammatory conditions.

For example, recent studies indicate that alveolar epithelial cells communicate with alveolar macrophages through connexins via calcium spikes during LPS-induced inflammation. ${ }^{113}$ This communication activates Akt-dependent signal propagation through adjacent cells, reducing neutrophil chemotaxis and proinflammatory cytokine release. These counterbalance proinflammatory responses in the lung, attenuating immunopathology. Similarly, the mucin MUC1 is induced by epithelial exposure to TNF and IL- $8,{ }^{114}$ and can regulate inflammation during bacterial infections through inhibition of TLR signaling. ${ }^{115,116}$ While MUC1-deficient mice show enhanced $P$. aeruginosa clearance following intranasal challenge, they also exhibit higher inflammatory chemokine expression, and greater neutrophil influx, resulting in greater immunopathology. ${ }^{117}$

The lungs also mitigate immunopathology via inhibitory feedback from the antimicrobial effector molecules described above. For example, epithelium-derived LL-37 can sequester LPS $^{72}$ and decrease cytokine release ${ }^{118}$ to dampen immune responses. Similarly, epithelial $\alpha$-defensin 1 can actively attenuate phagocyte ROS burst during influenza infections without impairing viral resistance. ${ }^{119}$ Further preventing immunopathology, epithelial cells produce mediators that promote resolution of inflammation, induce apoptosis, and decrease neutrophil chemotaxis. ${ }^{120,121}$ Polyunsaturated fatty acids present in epithelial cell membranes can be enzymatically converted to resolvins, maresins, protectins, and lipoxins. These molecules decrease proinflammatory cytokine expression, attenuate neutrophil oxidative burst, and decrease leukocyte chemotaxis, although their therapeutic inducibility remains largely untested.

\section{THERAPEUTIC MANIPULATION OF EPITHELIAL IMMUNITY}

The inducibility of the aforementioned epithelial defenses has prompted several groups to investigate exploitation of these mechanisms to protect against pneumonia. This approach offers an appealing complement to conventional antibiotic treatment, due to the lack of documented antibiotic resistance and broad protection that generally does not require prior pathogen identification. The most commonly applied strategies are exposure of lung cells to synthetic PRR ligands and the exogenous administration of AMPs (Table 3).

\section{Targeting of PRRs}

Early attempts to therapeutically harness inducible lung epithelial antimicrobial responses often centered on efforts to broadly stimulate PRRs by simulating native infections prior to experimental challenges. ${ }^{3}$ Typical of this nontargeted approach to PRR stimulation was intrapulmonary treatment of mice or isolated lung cells with noncognate bacterial lysates before pneumonia challenge. Exposing mice to the myriad PAMPs in a bacterial lysate induces robust responses that were shown to be protective against a wide range of otherwise lethal pathogens, including B. anthracis, Yersinia pestis, Francisella tularensis, S. pneumoniae, $P$. aeruginosa, S. aureus, $K$. pneumoniae, influenza A, and A. fumigatus. ${ }^{62,122}$ The protection was uniformly associated with pathogen killing in the lungs, and noted to correlate with enrichment of numerous antimicrobial molecules such as cathelicidin, defensins, lipocalin 2, surfactant proteins, orosomucoids, and calprotectins, ${ }^{62}$ suggesting activation of many of the above described processes. While treatment with crude lysates neither answered the questions of which PRRs were required for protection nor leant itself particularly well to clinical development, the finding that inducible protection was completely lost in MyD88-deficient mice $^{123}$ suggested that focused targeting of specific PRR population might be successful.

\section{TLR stimulation}

A substantial majority of studies investigating targeted PRR stimulation in the lungs have focused on manipulation of TLRs. The broad array of TLRs expressed by airway cells makes intrapulmonary administration of TLR agonists a conceptually attractive means of inducing resistance. Since shortly after the discovery of mammalian TLRs, investigators have delivered TLR agonists systemically to animals to initiate or modify leukocyte-mediated immunity, including efforts attempting to elicit secondary antimicrobial responses from intestinal epithelial cells. However, the presence of TLRs at the accessible environmental interface of the lungs allows for direct action on epithelial cells, which is a fundamentally different strategy than eliciting responses from circulating or intraepithelial leukocytes that then act on epithelial cells. In many cases, TLR ligands previously used to study signaling pathways have been repurposed for intrapulmonary delivery, with some ligands modified and/or conjugated to improve bioavailability and pharmacokinetics. For instance, intranasal delivery of a purified TLR5 agonist (flagellin) to the lungs protected mice against otherwise lethal $P$. aeruginosa pneumonia. This protection was partially dependent upon cathelicidin induction and persisted when neutrophils were depleted. ${ }^{124}$ A related strategy delivering intranasal pretreatment with an albuminconjugated TLR7 agonist (UC-1V150) protected mice against pneumonia caused by $B$. anthracis or $\mathrm{H} 1 \mathrm{~N} 1$ influenza. This 
Table 3 Targeted therapies for innate immunity in lung infections

\begin{tabular}{|c|c|c|c|c|c|}
\hline Component & $\begin{array}{l}\text { Receptor/ } \\
\text { ligand }\end{array}$ & Route & Protects against & Presumed mechanism & Reference \\
\hline \multicolumn{6}{|l|}{ Pattern-recognition receptors } \\
\hline UC-1V150 & TLR7 & I.T. & B. anthracis, influenza H1N1, VEE & IFN induction & 126 \\
\hline $3 M-011$ & $\begin{array}{l}\text { TLR7 and } \\
\text { TLR8 }\end{array}$ & I.N. & Influenza virus & IFN $\alpha$ induction & 129 \\
\hline PO R10-60 & TLR9 & I.N. & B. anthracis & & 130 \\
\hline Poly (I:C) & TLR3 & I.N. & $P$. aeruginosa, $F$. tularensis, influenza A & Chemokine induction & 127 \\
\hline Pam2CSK4 + ODNM62 & $\begin{array}{l}\text { TLR2/6 and } \\
\text { TLR9 }\end{array}$ & Nebulized & $\begin{array}{l}\text { S. pneumoniae, } P \text {. aeruginosa, B. anthracis, } \\
\text { influenza A, A. fumigatus }\end{array}$ & AMP and ROS generation & $123,132-134,155$ \\
\hline Flagellin & TLR5 & I.N. & S. pneumoniae and influenza A & Neutrophil recruitment & 156 \\
\hline 5'pppRNA & RIG-I & I.V. & VSV, Dengue, Vaccinia, HIV, H1N1 influenza & Type 1 IFN induction & 135,139 \\
\hline Eritoran (E5564) & $\begin{array}{l}\text { TLR4 } \\
\text { antagonist }\end{array}$ & I.V. & Influenza A & $\begin{array}{l}\text { Proinflammatory cytokine } \\
\text { blockade }\end{array}$ & 136 \\
\hline AGP & TLR4 & I.N. & F. novicida & IFN $\gamma$ induction & 131 \\
\hline CpG ODN & TLR9 & І.T. & K. pneumonie & $\begin{array}{l}\text { IFN } \gamma \text { and chemokine } \\
\text { induction }\end{array}$ & 157 \\
\hline
\end{tabular}

Antimicrobial Peptides

\begin{tabular}{llll} 
LL-37 & I.T. & P. aeruginosa, MRSA, RSV, M. tuberculosis & Membrane disruption \\
CAP18 & I.T. & P. aeruginosa & Chemokine induction \\
L-isoleucine & I.T. & Multidrug-resistant M. tuberculosis & HBD-2 induction \\
Novospirin G10 & I.T. & P. aeruginosa & Direct microbicidal \\
$\beta$-defensin 2 & I.T. & P. aeruginosa & HBD-2 upregulation \\
\hline
\end{tabular}

AGP, amynoalkyl-glucosamine phosphate; AMP, antimicrobial peptide; HBD, human beta defensin; IFN, interferon; I.N., intranasal; I.T., intratracheal; I.V., intravenous; MRSA, methicillin-resistant Staphylococcus aureus; ROS, reactive oxygen species; RSV, respiratory syncytial virus; TLR, Toll-like receptor; VEE, venezuelan equine encephalitis virus; VSV, vesicular stomatitis virus.

enhanced survival correlated with increased airway cytokine and chemokine production. ${ }^{125,126}$ Similarly, intranasal administration of a TLR3 agonist (poly I:C) during a cecal ligation and puncture sepsis model enhanced survival and decreased lung bacterial burdens following a secondary $P$. aeruginosa pneumonia challenge. ${ }^{127}$ Prophylactic or post-exposure intranasal poly I:C treatment also enhanced survival of $F$. tularensis pneumonia in a manner that was associated with increased local cytokine secretion and neutrophil influx to the airway, along with decreased lung pathogen burden. ${ }^{128}$ Another study of intranasal pretreatment of the lungs with a TLR7/8 agonist (3M-011) enhanced H3N2 influenza clearance in a rat model of pneumonia, noting the viral titer reductions to correlate with increases in TNF, IL-12 p40/70, and type I IFNs. ${ }^{129}$ Intranasal prophylactic TLR9 stimulation with a phosphodiester-oligodeoxynucleotide (PO R10-60) also protected mice against $B$. anthracis pneumonia, and increased inflammatory cytokines and type I interferons in lavage fluid. ${ }^{130}$ In a model of pneumonic tularemia, mice treated with the synthetic TLR4 ligand aminoalkyl glucosaminide phosphate before and after infection displayed increased survival. This protection was dependent on neutrophil influx and cytokine production in the airway and was lost in IFN $\gamma$ knockout mice. ${ }^{131}$

The broad protection induced by nontargeted stimuli (e.g., bacterial lysates), the robust responses generated by intrapulmonary application of specific ligands, and the positive microbicidal interactions observed when combining antimicrobial effector molecules together suggest that combinations of TLR stimuli might confer greater pneumonia protection than achieved with a single ligand. This hypothesis is supported by studies such as those testing concurrent inhalational administration of a TLR2/6 ligand (Pam2CSK4) and a TLR9 ligand (ODN M362) that synergistically interact to robustly protect mice against viral, bacterial and fungal pneumonia. ${ }^{132,133}$ Protection induced by the synergistic inhaled TLR ligands is dependent upon lung epithelial TLR signaling and is associated with production of both ROS and AMPs. ${ }^{134}$

Notably, all of the TLR agonist treatments described above initiate local inflammatory responses, and the reported survival benefits are all associated with reduced lung pathogen burdens, suggesting generation of a microbicidal environment. Further, all of the foregoing TLR stimulation strategies are presumed to predominantly engage lung epithelial TLRs, based on their delivery to the airways via intranasal, intratracheal or inhalational exposure. While epithelial cells comprise the vast majority of the treated surface area, TLRs on other cells (particularly, leukocytes) also encounter the ligands and likely generate relevant responses, as well. Thus, although the epithelial TLR requirement is established in these various treatments by loss of protection when TLR signaling is 
conditionally disrupted in the lung epithelium and/or by recapitulation of the antimicrobial phenomena when epithelial cells are studied in isolation, ${ }^{122,125,127,130,134,135}$ in vivo sufficiency of the epithelium to effect the complete phenotype is seldom established.

\section{TLR inhibition}

The immunopathology caused by excessive inflammation induced by certain infections may exceed the injury caused by pathogen virulence factors. In these cases, induction of tolerance may be a better strategy to survive infection. Mice lacking TLR4 seem to have an increased tolerance to influenza and inhibition of TLR4 signaling with eritoran 2 days after established infection decreased viral titers, alveolar inflammation, cytokine secretion, and overall survival. ${ }^{136}$ Although this strategy has been investigated in the clinical context of sepsis, it has not been demonstrated to improve overall mortality and it risks rendering the host susceptible to death by secondary bacterial pneumonia, ${ }^{137}$ potentially limiting its current applicability.

\section{RIG-I stimulation}

A synthetic 5'-triphosphate RNA RIG-I ligand (M8) has been shown to initiate protective responses against viral infections. Similar to several synthetic TLR agonists, M8 induces antiviral responses from isolated A549 lung epithelial cells against both RNA and DNA viruses. However, although in vitro epithelial responses have been demonstrated and intravenous injection of M8 before challenge with H1N1 influenza increased survival and decreased viral titers in the lung, ${ }^{138,139}$ there remain no in vivo data following direct intrapulmonary administration.

\section{EFFECTOR MOLECULE SUPPLEMENTATION \\ Antimicrobial peptides}

In vitro investigations using synthetic small cationic AMPs have long offered promise as microbicidal interventions, although the ion charges and concentrations used in pathogen killing experiments may be meaningfully different from in vivo conditions. In fact, survival benefit following in vivo administration of cationic peptides appears to largely rely on their ability to modulate inflammatory responses in the lungs, with rather inconsistent antimicrobial effects. ${ }^{140,141}$ For example, while it was found that synthetic human LL-37 instilled intranasally during $P$. aeruginosa infection decreased the pulmonary pathogen burden at $24 \mathrm{~h}$, there was no bacterial difference at earlier time points and enhanced survival appeared to correlate more strongly with neutrophil chemotactic properties of LL-37. ${ }^{142}$ This is concordant with other reports that LL-37 instillation promotes recruitment of neutrophils and monocytes. ${ }^{143}$ Intratracheal delivery of a related rabbit-derived molecule cationic antimicrobial peptide 18 (CAP18) also enhanced mouse survival of $P$. aeruginosa pneumonia. Similar to LL-37, the survival advantage was not associated with differences in bacterial burdens, but there was a significant decrease in proinflammatory interleukins in lavage fluid. ${ }^{118}$ Human beta-defensin-2 is a highly inducible airway epithelial AMP. Overexpression of human beta-defensin-2 before intratracheal $P$. aeruginosa infection, attenuates lung inflammation and a significantly increases survival. ${ }^{144}$ Similarly, in a pulmonary multidrug-resistant $M$. tuberculosis model, intratracheal administration of L-isoleucine 60 days after infection significantly increased mBD-3 (the murine ortholog of human beta-defensin-2). This increase was associated with a decreased bacterial burdens and pneumonia extent, ${ }^{145}$ as well as increased levels of lung TNF and IFN $\gamma$. Novispirin G10, a cationic protein isolated from sheep neutrophils, delivered to rats intratracheally immediately after infection with $P$. aeruginosa significantly decreased bacterial burdens and lung damage compared to sham treatment, although no survival difference was noted. ${ }^{146}$ IL-22 which can upregulate lipocalin- 2 has been shown to rescue IL-23 deficient mice in the setting of acute $K$. pneumoniae infection. ${ }^{88}$ The lung epithelium also expresses members of the regenerating isletderived proteins which can bind peptidoglycan on grampositive bacteria. ${ }^{147}$ These proteins are STAT3-regulated and undergo post-translational modification in the lung to exert bactericidal activity against MRSA. ${ }^{148}$ Collectively, these examples demonstrate that therapeutically supplemented AMPs frequently elicit effects by both microbicidal and immunomodulatory mechanisms, just as observed with native AMP induction.

Relatedly, cytokine supplementation has been undertaken with the intent of modulating immune responses, but as discussed above, several of these molecules also possess antimicrobial activity. Thus, their supplementation may directly reduce pathogen burden, as well.

\section{ALTERNATE STRATEGIES}

Although induction of epithelial ROS can kill pathogens, direct supplementation of ROS or other volatiles to the lung is generally not feasible. However, dietary supplementation or nebulized delivery of halide and pseudohalide substrates to the lung epithelium can enhance peroxidase-catalyzed production of highly microbicidal, ROS-dependent molecules (e.g., hypothiocyanate, hypoiodous acid, hypochlorous acid). To date, these studies have been associated with increases in the investigated antimicrobial molecules and reductions in pathogen burdens, ${ }^{99,149,150}$ but have not demonstrated enhanced pneumonia survival.

Another epithelium-targeted strategy to protect against pneumonia is the potentiation of antimicrobial and mucociliary function through manipulation of periciliary $\mathrm{pH}$. In piglets with cystic fibrosis, inhalation of bicarbonate or tromethamine increases periciliary $\mathrm{pH}$, enhancing the bactericidal function of AMPs. ${ }^{151}$ This may explain the decrease in $P$. aeruginosa colonization in patients treated with the CFTR potentiator ivacaftor. ${ }^{152,153}$ Reports describing successful manipulation of barrier function or induction of pneumonia tolerance remain lacking. However, TLR5 stimulation has been shown to reduce immunopathology in other conditions, such as radiation injury, ${ }^{154}$ so targeting these elements of epithelial defense may be feasible. 


\section{CONCLUSIONS}

Taken together, the lung epithelium is capable of significant antimicrobial responses and actively participates in different threat-reduction strategies against pathogen load and noxious stimuli. It is possible to take advantage of these mechanisms to prevent infection during peak susceptibility periods despite leukocyte dysfunction or depletion. Further research in mucosal innate immunity will provide insight into potential targeted therapies for induction of epithelial antimicrobial responses and prevention of lung injury in humans.

\section{ACKNOWLEDGMENTS}

This work was supported in part by National Institutes of Health grants R01 HL117976 and DP2 HL123229 to S.E.E.

\section{DISCLOSURE}

M.M.L.-J. declares no conflicts of interest.J.K.K. declares no conflicts of interest. S.E.E. is an author on US patent 8,883,174 entitled 'Stimulation of Innate Resistance of the Lungs to Infection with Synthetic Ligands' and owns stock in Pulmotect, Inc., which holds the commercial options on these patent disclosures.

c) 2018 Society for Mucosal Immunology

\section{REFERENCES}

1. Dickson, R.P. \& Huffnagle, G.B. The lung microbiome: new principles for respiratory bacteriology in health and disease. PLoS Pathog. 11, e1004923 (2015)

2. Whitsett, J.A. \& Alenghat, T. Respiratory epithelial cells orchestrate pulmonary innate immunity. Nat. Immunol. 16, 27-35 (2015).

3. Evans, S.E., Xu, Y., Tuvim, M.J. \& Dickey, B.F. Inducible innate resistance of lung epithelium to infection. Annu. Rev. Physiol. 72, 413-435 (2010).

4. Franks, T.J. et al. Resident cellular components of the human lung: current knowledge and goals for research on cell phenotyping and function. Proc. Am. Thorac. Soc. 5, 763-766 (2008).

5. He, D. et al. Lysophosphatidic acid enhances pulmonary epithelial barrier integrity and protects endotoxin-induced epithelial barrier disruption and lung injury. J. Biol. Chem. 284, 24123-24132 (2009).

6. Wray, C. et al. Claudin-4 augments alveolar epithelial barrier function and is induced in acute lung injury. Am. J. Physiol. Lung Cell. Mol. Physiol. 297. L219-L227 (2009).

7. Davies, D.E. Epithelial barrier function and immunity in asthma. Ann. Am. Thorac. Soc. 11 (Suppl 5), S244-S251 (2014).

8. Brune, K., Frank, J., Schwingshackl, A., Finigan, J. \& Sidhaye, V.K. Pulmonary epithelial barrier function: some new players and mechanisms. Am. J. Physiol. Lung Cell. Mol. Physiol. 308, L731-L745 (2015).

9. Ragupathy, S. et al. Toll-like receptor 2 regulates the barrier function of human bronchial epithelial monolayers through atypical protein kinase $\mathrm{C}$ zeta, and an increase in expression of claudin-1. Tissue Barriers 2, e29166 (2014).

10. Sancho, D. et al. Identification of a dendritic cell receptor that couples sensing of necrosis to immunity. Nature 458, 899-903 (2009).

11. Mostowy, S. et al. Entrapment of intracytosolic bacteria by septin cage-like structures. Cell Host Microbe 8, 433-444 (2010).

12. Roy, M.G. et al. Muc5b is required for airway defence. Nature 505, 412-416 (2014).

13. Fahy, J.V. \& Dickey, B.F. Airway mucus function and dysfunction. N. Engl. J. Med. 363, 2233-2247 (2010).

14. Kim, Y.D., Bae, C.H., Song, S.Y. \& Choi, Y.S. Effect of $\beta$-glucan on MUC4 and MUC5B expression in human airway epithelial cells. Int. Forum Allergy Rhinol. 5, 708-715 (2015).

15. Fujisawa, T. et al. NF-kB mediates IL-1 $\beta$ - and IL-17 A-induced MUC5B expression in airway epithelial cells. Am. J. Respir. Cell Mol. Biol. 45, 246-252 (2011).

16. Voynow, J.A. \& Rubin, B.K. Mucins, mucus, and sputum. Chest $\mathbf{1 3 5}$ 505-512 (2009).
17. Zhang, Y. et al. The human Cathelicidin LL-37 induces MUC5AC mucin production by airway epithelial cells via TACE-TGF- $\alpha$-EGFR pathway. Exp. Lung Res. 40, 333-342 (2014).

18. Holtzman, M.J. et al. Immune pathways for translating viral infection into chronic airway disease. Adv. Immunol. 102, 245-276 (2009).

19. Barbier, D. et al. Influenza A induces the major secreted airway mucin MUC5AC in a protease-EGFR-extracellular regulated kinase-Sp1dependent pathway. Am. J. Respir. Cell Mol. Biol. 47, 149-157 (2012).

20. Wlodarska, M. et al. NLRP6 inflammasome orchestrates the colonic hostmicrobial interface by regulating goblet cell mucus secretion. Cell 156, 1045-1059 (2014).

21. Hoegger, M.J. et al. Impaired mucus detachment disrupts mucociliary transport in a piglet model of cystic fibrosis. Science 345, 818-822 (2014).

22. Bonser, L.R., Zlock, L., Finkbeiner, W. \& Erle, D.J. Epithelial tethering of MUC5AC-rich mucus impairs mucociliary transport in asthma. J. Clin. Invest. 126, 2367-2371 (2016).

23. Roach, J.C. et al. The evolution of vertebrate Toll-like receptors. Proc. Natl Acad. Sci. USA 102, 9577-9582 (2005).

24. Burch-Smith, T.M. \& Dinesh-Kumar, S.P. The functions of plant TIR domains. SCi. STKE 2007, pe46 (2007).

25. Lemaitre, B., Nicolas, E., Michaut, L., Reichhart, J.M. \& Hoffmann, J.A. The dorsoventral regulatory gene cassette spätzle/Toll/cactus controls the potent antifungal response in Drosophila adults. Cell 86, 973-983 (1996).

26. Gay, N.J., Symmons, M.F., Gangloff, M. \& Bryant, C.E. Assembly and Iocalization of Toll-like receptor signalling complexes. Nat. Rev. Immunol. 14, 546-558 (2014).

27. Botos, I., Segal, D.M. \& Davies, D.R. The structural biology of Toll-like receptors. Structure 19, 447-459 (2011).

28. Chaput, C., Sander, L.E., Suttorp, N. \& Opitz, B. NOD-like receptors in lung diseases. Front. Immunol. 4, 393 (2013).

29. Broz, P. \& Monack, D.M. Newly described pattern recognition receptors team up against intracellular pathogens. Nat. Rev. Immunol. 13, 551-565 (2013).

30. Divangahi, M. et al. NOD2-deficient mice have impaired resistance to Mycobacterium tuberculosis infection through defective innate and adaptive immunity. J. Immunol. 181, 7157-7165 (2008).

31. Austin, C.M., Ma, X. \& Graviss, E.A. Common nonsynonymous polymorphisms in the NOD2 gene are associated with resistance or susceptibility to tuberculosis disease in African Americans. J. Infect. Dis. 197, 1713-1716 (2008).

32. Chavarría-Smith, J. \& Vance, R.E. Direct proteolytic cleavage of NLRP1B is necessary and sufficient for inflammasome activation by anthrax lethal factor. PLoS Pathog. 9, e1003452 (2013).

33. De Nardo, D., De Nardo, C.M. \& Latz, E. New insights into mechanisms controlling the NLRP3 inflammasome and its role in lung disease. Am. J. Pathol. 184, 42-54 (2014).

34. Kostadinova, E. et al. NLRP3 protects alveolar barrier integrity by an inflammasome-independent increase of epithelial cell adherence. Sci. Rep. 6, 30943 (2016).

35. Pétrilli, V. et al. Activation of the NALP3 inflammasome is triggered by low intracellular potassium concentration. Cell Death Differ. 14, 1583-1589 (2007).

36. Dostert, C. et al. Innate immune activation through Nalp3 inflammasome sensing of asbestos and silica. Science 320, 674-677 (2008).

37. Zhou, R., Yazdi, A.S., Menu, P. \& Tschopp, J. A role for mitochondria in NLRP3 inflammasome activation. Nature 469, 221-225 (2011).

38. Berrington, W.R., Smith, K.D., Skerrett, S.J. \& Hawn, T.R. Nucleotidebinding oligomerization domain containing-like receptor family, caspase recruitment domain (CARD) containing 4 (NLRC4) regulates intrapulmonary replication of aerosolized Legionella pneumophila. BMC Infect. Dis. 13, 371 (2013).

39. Tolle, L. et al. Redundant and cooperative interactions between TLR5 and NLRC4 in protective lung mucosal immunity against Pseudomonas aeruginosa. J. Innate Immun. 7, 177-186 (2015).

40. Schneider, M. et al. The innate immune sensor NLRC3 attenuates Toll-like receptor signaling via modification of the signaling adaptor TRAF6 and transcription factor NF-кB. Nat. Immunol. 13, 823-831 (2012).

41. Sharma, S. \& Fitzgerald, K.A. Innate immune sensing of DNA. PLoS Pathog. 7, e1001310 (2011). 
42. Opitz, B., van Laak, V., Eitel, J. \& Suttorp, N. Innate immune recognition in infectious and noninfectious diseases of the lung. Am. J. Respir. Crit. Care Med. 181, 1294-1309 (2010).

43. Bhoj, V.G. et al. MAVS and MyD88 are essential for innate immunity but not cytotoxic $T$ lymphocyte response against respiratory syncytial virus. Proc. Natl Acad. Sci. USA 105, 14046-14051 (2008).

44. Foronjy, R.F. et al. Type-I interferons induce lung protease responses following respiratory syncytial virus infection via RIG-I-like receptors. Mucosal Immunol. 8, 161-175 (2015).

45. Kato, $\mathrm{H}$. et al. Differential roles of MDA5 and RIG-I helicases in the recognition of RNA viruses. Nature 441, 101-105 (2006).

46. Ishikawa, H. \& Barber, G.N. STING is an endoplasmic reticulum adaptor that facilitates innate immune signalling. Nature 455, 674-678 (2008).

47. Burdette, D.L. \& Vance, R.E. STING and the innate immune response to nucleic acids in the cytosol. Nat. Immunol. 14, 19-26 (2013).

48. Unterholzner, L. et al. IFl16 is an innate immune sensor for intracellular DNA. Nat. Immunol. 11, 997-1004 (2010).

49. Zhang, Z. etal. The helicase DDX41 senses intracellular DNA mediated by the adaptor STING in dendritic cells. Nat. Immunol. 12, 959-965 (2011).

50. Parvatiyar, K. et al. The helicase DDX41 recognizes the bacterial secondary messengers cyclic di-GMP and cyclic di-AMP to activate a type I interferon immune response. Nat. Immunol. 13, 1155-1161 (2012).

51. Wu, J. et al. Cyclic GMP-AMP is an endogenous second messenger in innate immune signaling by cytosolic DNA. Science 339, 826-830 (2013).

52. Yang, P. et al. The cytosolic nucleic acid sensor LRRFIP1 mediates the production of type I interferon via a beta-catenin-dependent pathway. Nat. Immunol. 11, 487-494 (2010).

53. Geijtenbeek, T.B. \& Gringhuis, S.I. Signalling through C-type lectin receptors: shaping immune responses. Nat. Rev. Immunol. 9, 465-479 (2009).

54. Heyl, K.A. et al. Dectin-1 is expressed in human lung and mediates the proinflammatory immune response to nontypeable Haemophilus influenzae. MBio 5, e01492-01414 (2014).

55. Drummond, R.A. \& Lionakis, M.S. Mechanistic insights into the role of C-Type lectin receptor/CARD9 signaling in human antifungal immunity. Front. Cell. Infect. Microbiol. 6, 39 (2016).

56. Nayak, A., Dodagatta-Marri, E., Tsolaki, A.G. \& Kishore, U. An insight into the diverse roles of surfactant proteins, SP-A and SP-D in innate and adaptive immunity. Front. Immunol. 3, 131 (2012).

57. Evans, S.E. et al. Pneumocystis cell wall beta-glucans stimulate alveolar epithelial cell chemokine generation through nuclear factor-kappaBdependent mechanisms. Am. J. Respir. Cell Mol. Biol. 32, 490-497 (2005).

58. Kolls, J.K., McCray, P.B. \& Chan, Y.R. Cytokine-mediated regulation of antimicrobial proteins. Nat. Rev. Immunol. 8, 829-835 (2008).

59. Ganz, T. Defensins: antimicrobial peptides of innate immunity. Nat. Rev. Immunol. 3, 710-720 (2003).

60. Lecaille, F., Lalmanach, G. \& Andrault, P.M. Antimicrobial proteins and peptides in human lung diseases: a friend and foe partnership with host proteases. Biochimie 122, 151-168 (2015).

61. Frye, M. etal. Expression of human alpha-defensin 5 (HD5) mRNA in nasal and bronchial epithelial cells. J. Clin. Pathol. 53, 770-773 (2000).

62. Evans, S.E. et al. Stimulated innate resistance of lung epithelium protects mice broadly against bacteria and fungi. Am. J. Respir. Cell Mol. Biol. 42, 40-50 (2010).

63. Zanetti, M. Cathelicidins, multifunctional peptides of the innate immunity. J. Leukoc. Biol. 75, 39-48 (2004).

64. Bals, R., Wang, X., Zasloff, M. \& Wilson, J.M. The peptide antibiotic LL-37/ hCAP-18 is expressed in epithelia of the human lung where it has broad antimicrobial activity at the airway surface. Proc. Natl Acad. Sci. USA 95, 9541-9546 (1998)

65. Akiyama, T. et al. The human cathelicidin LL-37 host defense peptide upregulates tight junction-related proteins and increases human epidermal keratinocyte barrier function. J. Innate Immun. 6, 739-753 (2014).

66. De Yang, et al. LL-37, the neutrophil granule- and epithelial cell-derived cathelicidin, utilizes formyl peptide receptor-like 1 (FPRL1) as a receptor to chemoattract human peripheral blood neutrophils, monocytes, and T cells. J. Exp. Med. 192, 1069-1074 (2000).

67. Yang, D. et al. Beta-defensins: linking innate and adaptive immunity through dendritic and T cell CCR6. Science 286, 525-528 (1999).
68. Zhang, Z. et al. Evidence that cathelicidin peptide LL-37 may act as a functional ligand for CXCR2 on human neutrophils. Eur. J. Immunol. 39, 3181-3194 (2009).

69. Yu, J. et al. Host defense peptide LL-37, in synergy with inflammatory mediator IL-1 beta, augments immune responses by multiple pathways. J. Immunol. 179, 7684-7691 (2007).

70. Ganguly, D. et al. Self-RNA-antimicrobial peptide complexes activate human dendritic cells through TLR7 and TLR8. J. Exp. Med. 206, 1983-1994 (2009).

71. Lande, R. et al. Plasmacytoid dendritic cells sense self-DNA coupled with antimicrobial peptide. Nature 449, 564-569 (2007).

72. Mookherjee, N. et al. Modulation of the TLR-mediated inflammatory response by the endogenous human host defense peptide LL-37. J. Immunol. 176, 2455-2464 (2006).

73. Williams, S.E., Brown, T.I., Roghanian, A. \& Sallenave, J.M. SLPI and elafin: one glove, many fingers. Clin. Sci. (Lond.) 110, 21-35 (2006).

74. Tomee, J.F., Hiemstra, P.S., Heinzel-Wieland, R. \& Kauffman, H.F. Antileukoprotease: an endogenous protein in the innate mucosal defense against fungi. J. Infect. Dis. 176, 740-747 (1997).

75. Hiemstra, P.S. et al. Antibacterial activity of antileukoprotease. Infect. Immun. 64, 4520-4524 (1996).

76. Simpson, A.J., Maxwell, A.I., Govan, J.R., Haslett, C. \& Sallenave, J.M. Elafin (elastase-specific inhibitor) has anti-microbial activity against gram-positive and gram-negative respiratory pathogens. FEBS Lett. 452, 309-313 (1999).

77. Henriksen, P.A. et al. Adenoviral gene delivery of elafin and secretory leukocyte protease inhibitor attenuates NF-kappa B-dependent inflammatory responses of human endothelial cells and macrophages to atherogenic stimuli. J. Immunol. 172, 4535-4544 (2004).

78. Sano, C., Shimizu, T., Sato, K., Kawauchi, H. \& Tomioka, H. Effects of secretory leucocyte protease inhibitor on the production of the anti-inflammatory cytokines, IL-10 and transforming growth factor-beta (TGF-beta), by lipopolysaccharide-stimulated macrophages. Clin. Exp. Immunol. 121, 77-85 (2000).

79. van Bergen, B.H., Andriessen, M.P., Spruijt, K.I., van de Kerkhof, P.C. \& Schalkwijk, J. Expression of SKALP/elafin during wound healing in human skin. Arch. Dermatol. Res. 288, 458-462 (1996).

80. Margulieux, K.R., Fox, J.W., Nakamoto, R.K. \& Hughes, M.A. CXCL10 acts as a bifunctional antimicrobial molecule against Bacillus anthracis. MBio 7, (2016).

81. Cole, A.M. et al. Cutting edge: IFN-inducible ELR- CXC chemokines display defensin-like antimicrobial activity. J. Immunol. 167, 623-627 (2001).

82. Dai, C. et al. CXCL14 displays antimicrobial activity against respiratory tract bacteria and contributes to clearance of Streptococcus pneumoniae pulmonary infection. J. Immunol. 194, 5980-5989 (2015).

83. Dajani, R. et al. Lysozyme secretion by submucosal glands protects the airway from bacterial infection. Am. J. Respir. Cell Mol. Biol. 32, 548-552 (2005).

84. Akinbi, H.T., Epaud, R., Bhatt, H. \& Weaver, T.E. Bacterial killing is enhanced by expression of lysozyme in the lungs of transgenic mice. J. Immunol. 165, 5760-5766 (2000).

85. Ellison, R.T. 3rd \& Giehl, T.J. Killing of gram-negative bacteria by lactoferrin and lysozyme. J. Clin. Invest. 88, 1080-1091 (1991).

86. Flo, T.H. et al. Lipocalin 2 mediates an innate immune response to bacterial infection by sequestrating iron. Nature 432, 917-921 (2004).

87. Chan, Y.R. et al. Lipocalin 2 is required for pulmonary host defense against Klebsiella infection. J. Immunol. 182, 4947-4956 (2009).

88. Aujla, S.J. et al. IL-22 mediates mucosal host defense against Gram-negative bacterial pneumonia. Nat. Med. 14, 275-281 (2008).

89. Crouch, E.C. Collectins and pulmonary host defense. Am. Respir. Cell. Mol. Biol. 19, 177-201 (1998).

90. LeVine, A.M. \& Whitsett, J.A. Pulmonary collectins and innate host defense of the lung. Microbes Infect. 3, 161-166 (2001).

91. $\mathrm{Wu}, \mathrm{H}$. et al. Surfactant proteins $A$ and $D$ inhibit the growth of Gram-negative bacteria by increasing membrane permeability. J. Clin. Invest 111, 1589-1602 (2003).

92. Thaikoottathil, J. \& Chu, H.W. MAPK/AP-1 activation mediates TLR2 agonist-induced SPLUNC1 expression in human lung epithelial cells. Mol Immunol 49, 415-422 (2011). 
93. Bingle, C.D. \& Gorr, S.U. Host defense in oral and airway epithelia: chromosome 20 contributes a new protein family. Int. J. Biochem. Cell Biol. 36, 2144-2152 (2004).

94. Liu, Y. et al. Increased susceptibility to pulmonary Pseudomonas infection in Splunc1 knockout mice. J. Immunol. 191, 4259-4268 (2013).

95. Liu, Y. et al. SPLUNC1/BPIFA1 contributes to pulmonary host defense against Klebsiella pneumoniae respiratory infection. Am. J. Pathol. 182, 1519-1531 (2013).

96. Fischer, H. Mechanisms and function of DUOX in epithelia of the lung. Antioxid. Redox Signal. 11, 2453-2465 (2009).

97. Forteza, R., Salathe, M., Miot, F. \& Conner, G.E. Regulated hydrogen peroxide production by Duox in human airway epithelial cells. Am. J. Respir. Cell Mol. Biol. 32, 462-469 (2005).

98. Pezzulo, A.A. et al. Reduced airway surface $\mathrm{pH}$ impairs bacterial killing in the porcine cystic fibrosis lung. Nature 487, 109-113 (2012).

99. Chandler, J.D. \& Day, B.J. Biochemical mechanisms and therapeutic potential of pseudohalide thiocyanate in human health. Free Radic. Res. 49, 695-710 (2015).

100. Hammad, H. et al. House dust mite allergen induces asthma via Toll-like receptor 4 triggering of airway structural cells. Nat. Med. 15, 410-416 (2009).

101. Allakhverdi, Z. et al. Thymic stromal lymphopoietin is released by human epithelial cells in response to microbes, trauma, or inflammation and potently activates mast cells. J Exp Med 204, 253-258 (2007).

102. Llop-Guevara, A. et al. A GM-CSF/L-33 pathway facilitates allergic airway responses to sub-threshold house dust mite exposure. PLoS One 9, e88714 (2014).

103. O'Grady, S.M. etal. ATP release and Ca2 + signalling by human bronchial epithelial cells following Alternaria aeroallergen exposure. J. Physiol. 591, 4595-4609 (2013).

104. Kool, M. et al. An unexpected role for uric acid as an inducer of Thelper 2 cell immunity to inhaled antigens and inflammatory mediator of allergic asthma. Immunity 34, 527-540 (2011).

105. Dulek, D.E. et al. Allergic airway inflammation decreases lung bacterial burden following acute Klebsiella pneumoniae infection in a neutrophiland CCL8-dependent manner. Infect. Immun. 82, 3723-3739 (2014).

106. Sharma, M., Sharma, S., Roy, S., Varma, S. \& Bose, M. Pulmonary epithelial cells are a source of interferon-gamma in response to Mycobacterium tuberculosis infection. Immunol. Cell Biol. 85, 229-237 (2007).

107. Murdock, B.J., Huffnagle, G.B., Olszewski, M.A. \& Osterholzer, J.J. Interleukin-17A enhances host defense against cryptococcal lung infection through effects mediated by leukocyte recruitment, activation, and gamma interferon production. Infect. Immun. 82, 937-948 (2014).

108. Lee, H.S. et al. IL-23 secreted by bronchial epithelial cells contributes to allergic sensitization in asthma model: role of IL-23 secreted by bronchial epithelial cells. Am. J. Physiol. Lung Cell. Mol. Physiol. 312, L13-L21 (2017).

109. Spolski, R. \& Leonard, W.J. Interleukin-21: a double-edged sword with therapeutic potential. Nat. Rev. Drug Discov. 13, 379-395 (2014).

110. Di Stefano, A. et al. T helper type 17-related cytokine expression is increased in the bronchial mucosa of stable chronic obstructive pulmonary disease patients. Clin. Exp. Immunol. 157, 316-324 (2009).

111. Ouyang, W., Kolls, J.K. \& Zheng, Y. The biological functions of Thelper 17 cell effector cytokines in inflammation. Immunity 28, 454-467 (2008).

112. Schneider, D.S. \& Ayres, J.S. Two ways to survive infection: what resistance and tolerance can teach us about treating infectious diseases. Nat. Rev. Immunol. 8, 889-895 (2008).

113. Westphalen, K. et al. Sessile alveolar macrophages communicate with alveolar epithelium to modulate immunity. Nature 506, 503-506 (2014).

114. Koga, T. et al. TNF-alpha induces MUC1 gene transcription in lung epithelial cells: its signaling pathway and biological implication. Am. J. Physiol. Lung Cell. Mol. Physiol. 293, L693-L701 (2007).

115. Ueno, K. et al. MUC1 mucin is a negative regulator of toll-like receptor signaling. Am. J. Respir. Cell. Mol. Biol. 38, 263-268 (2008).

116. Kim, K.C. \& Lillehoj, E.P. MUC1 mucin: a peacemaker in the lung. Am. J. Respir. Cell. Mol. Biol. 39, 644-647 (2008).

117. Lu, W. et al. Cutting edge: enhanced pulmonary clearance of Pseudomonas aeruginosa by Muc1 knockout mice. J. Immunol. 176, 3890-3894 (2006).
118. Sawa, T. et al. Evaluation of antimicrobial and lipopolysaccharideneutralizing effects of a synthetic CAP18 fragment against $P$ seudomonas aeruginosa in a mouse model. Antimicrob. Agents Chemother. 42, 3269-3275 (1998).

119. Tecle, T., White, M.R., Gantz, D., Crouch, E.C. \& Hartshorn, K.L. Human neutrophil defensins increase neutrophil uptake of influenza $A$ virus and bacteria and modify virus-induced respiratory burst responses. J. Immunol. 178, 8046-8052 (2007).

120. Uddin, M. \& Levy, B.D. Resolvins: natural agonists for resolution of pulmonary inflammation. Prog. Lipid Res. 50, 75-88 (2011).

121. Levy, B.D. \& Serhan, C.N. Resolution of acute inflammation in the lung Annu. Rev. Physiol. 76, 467-492 (2014).

122. Clement, C.G. et al. Stimulation of lung innate immunity protects against lethal pneumococcal pneumonia in mice. Am. J. Respir. Crit. Care Med. 177, 1322-1330 (2008).

123. Duggan, J.M. et al. Synergistic interactions of TLR2/6 and TLR9 induce a high level of resistance to lung infection in mice. J. Immunol. 186, 5916-5926 (2011).

124. Yu, F.S. et al. Flagellin stimulates protective lung mucosal immunity: role of cathelicidin-related antimicrobial peptide. J. Immunol. 185, 1142-1149 (2010).

125. Wu, C.C. et al. Innate immune protection against infectious diseases by pulmonary administration of a phospholipid-conjugated TLR7 ligand. J. Innate Immun. 6, 315-324 (2014).

126. Wu, C.C. et al. Immunotherapeutic activity of a conjugate of a Toll-like receptor 7 ligand. Proc. Natl Acad. Sci. USA 104, 3990-3995 (2007).

127. Davis, C.G. et al. TLR3 agonist improves survival to secondary pneumonia in a double injury model. J. Surg. Res. 182, 270-276 (2013).

128. Pyles, R.B., Jezek, G.E. \& Eaves-Pyles, T.D. Toll-like receptor 3 agonist protection against experimental Francisella tularensis respiratory tract infection. Infect. Immun. 78, 1700-1710 (2010).

129. Hammerbeck, D.M. et al. Administration of a dual toll-like receptor 7 and toll-like receptor 8 agonist protects against influenza in rats. Antiviral Res. 73, 1-11 (2007).

130. Wu, C.C. et al. In vivo efficacy of a phosphodiester TLR-9 aptamer and its beneficial effect in a pulmonary anthrax infection model. Cell. Immunol. 251, 78-85 (2008).

131. Lembo, A. et al. Administration of a synthetic TLR4 agonist protects mice from pneumonic tularemia. J. Immunol. 180, 7574-7581 (2008).

132. Tuvim, M.J., Gillbert, B.E., Dickey, B.F. \& Evans, S.E. Synergistic TLR2/6 and TLR9 activation protects mice against lethal influenza pneumonia. PLoS One 7, e30596 (2012).

133. Leiva-Juarez, M.M. et al. Inducible epithelial resistance protects mice against leukemia-associated pneumonia. Blood 128, 982-992 (2016).

134. Cleaver, J.O. et al. Lung epithelial cells are essential effectors of inducible resistance to pneumonia. Mucosal Immunol. 7, 78-88 (2014).

135. Goulet, M.L. et al. Systems analysis of a RIG-I agonist inducing broad spectrum inhibition of virus infectivity. PLoS Pathog. 9, e1003298 (2013).

136. Shirey, K.A. et al. The TLR4 antagonist Eritoran protects mice from lethal influenza infection. Nature 497, 498-502 (2013).

137. Morales-Nebreda, L., Mutlu, G.M., Scott Budinger, G.R. \& Radigan, K.A. Loss of TLR4 does not prevent influenza A-induced mortality. Am. J. Respir. Crit. Care Med. 189, 1280-1281 (2014).

138. Beljanski, V. et al. Enhanced influenza virus-like particle vaccination with a structurally optimized RIG-I agonist as adjuvant. J. Virol. 89, 10612-10624 (2015).

139. Chiang, C. et al. Sequence-specific modifications enhance the broad-spectrum antiviral response activated by RIG-I agonists. J. Virol. 89, 8011-8025 (2015).

140. Rivas-Santiago, B. et al. Activity of LL-37, CRAMP and antimicrobial peptide-derived compounds E2, E6 and CP26 against Mycobacterium tuberculosis. Int. J. Antimicrob. Agents 41, 143-148 (2013).

141. Bowdish, D.M., Davidson, D.J., Scott, M.G. \& Hancock, R.E. Immunomodulatory activities of small host defense peptides. Antimicrob. Agents Chemother. 49, 1727-1732 (2005).

142. Beaumont, P.E. et al. Cathelicidin host defence peptide augments clearance of pulmonary Pseudomonas aeruginosa infection by its influence on neutrophil function in vivo. PLoS One 9, e99029 (2014).

143. Kurosaka, K., Chen, Q., Yarovinsky, F., Oppenheim, J.J. \& Yang, D. Mouse cathelin-related antimicrobial peptide chemoattracts leukocytes 
using formyl peptide receptor-like 1 /mouse formyl peptide receptor-like 2 as the receptor and acts as an immune adjuvant. J. Immunol. 174, 6257-6265 (2005).

144. Shu, Q. et al. Protection against Pseudomonas aeruginosa pneumonia and sepsis-induced lung injury by overexpression of beta-defensin-2 in rats. Shock 26, 365-371 (2006).

145. Rivas-Santiago, C.E., Rivas-Santiago, B., León, D.A., CastañedaDelgado, J. \& Hernández Pando, R. Induction of $\beta$-defensins by I-isoleucine as novel immunotherapy in experimental murine tuberculosis. Clin. Exp. Immunol. 164, 80-89 (2011).

146. Song, Z. et al. Effects of intratracheal administration of novispirin $\mathrm{G} 10$ on a rat model of mucoid Pseudomonas aeruginosa lung infection. Antimicrob. Agents Chemother. 49, 3868-3874 (2005).

147. Mukherjee, S. et al. Antibacterial membrane attack by a pore-forming intestinal C-type lectin. Nature 505, 103-107 (2014).

148. Choi, S.M. et al. Innate Stat3-mediated induction of the antimicrobial protein Reg3 $\gamma$ is required for host defense against MRSA pneumonia. J. Exp. Med. 210, 551-561 (2013).

149. Chandler, J.D., Min, E., Huang, J., Nichols, D.P. \& Day, B.J. Nebulized thiocyanate improves lung infection outcomes in mice. Br. J. Pharmacol. 169, 1166-1177 (2013).

150. Fischer, A.J. et al. Enhancement of respiratory mucosal antiviral defenses by the oxidation of iodide. Am. J. Respir. Cell. Mol. Biol. 45, 874-881 (2011).
151. Abou Alaiwa, M.H. et al. Repurposing tromethamine as inhaled therapy to treat CF airway disease. JCl Insight 12016).

152. Heltshe, S.L. et al. Pseudomonas aeruginosa in cystic fibrosis patients with G551D-CFTR treated with ivacaftor. Clin. Infect. Dis. 60, 703-712 (2015).

153. Rowe, S.M. et al. Clinical mechanism of the cystic fibrosis transmembrane conductance regulator potentiator ivacaftor in G551D-mediated cystic fibrosis. Am. J. Respir. Crit. Care Med. 190, 175-184 (2014).

154. Burdelya, L.G. et al. An agonist of toll-like receptor 5 has radioprotective activity in mouse and primate models. Science 320, 226-230 (2008).

155. Alfaro, V.Y. et al. Safety, tolerability, and biomarkers of the treatment of mice with aerosolized Toll-like receptor ligands. Front. Pharmacol. 5, 8 (2014).

156. Porte, R. et al. A toll-like receptor 5 agonist improves the efficacy of antibiotics in treatment of primary and influenza virus-associated pneumococcal mouse infections. Antimicrob. Agents Chemother. 59, 6064-6072 (2015).

157. Deng, J.C. et al. CpG oligodeoxynucleotides stimulate protective innate immunity against pulmonary Klebsiella infection. J. Immunol. 173, 5148-5155 (2004).

158. Currie, S.M. et al. The human cathelicidin LL-37 has antiviral activity against respiratory syncytial virus. PLoS One 8, e73659 (2013). 